\title{
Subsets of Scales in Compositions Constructed by Similarity
}

\author{
Will Turner \\ Institute of Mathematics, University of Aberdeen, Aberdeen, Scotland
}

Email address:

w.turner@abdn.ac.uk

\section{To cite this article:}

Will Turner. Subsets of Scales in Compositions Constructed by Similarity. International Journal of Theoretical and Applied Mathematics. Vol. 7, No. 2, 2021, pp. 30-39. doi: 10.11648/j.ijtam.20210702.12

Received: April 14, 2021; Accepted: May 5, 2021; Published: May 27, 2021

\begin{abstract}
We present techniques in musical composition based on subsets of scales, built on theoretical notions, together with a number of examples. The techniques we describe are for constructing compositions with reference to memory, via similarity. We begin with some technical elements: after introducing the technique of intersecting accompaniments, we describe similarity concatenation compositions, which are special compositions constructed via similarity. We then outline a method to solve the problem of approximating scales with frequency ratios generated by rational numbers with small numerators and denominators, via equal temperament. As well as the standard solution via 12 tone equal temperament, we present a solution via 31 tone equal temperament. We then introduce the notion of a connected triheptad, generalising the tonic, subdominant and dominant of the major scale. We next present some examples of the notions previously introduced. Example 1 features a connected triheptad, and Example 2 features a connected triheptad, a similarity concatenation composition, and an intersecting accompaniment. There follows a section on cubist sets, featuring a returning similarity concatenation composition. We then move a conceptual level higher: we consider the concatenation of similarity concatenation compositions via similarity. This is reminiscent of higher dimensional algebra, and there follows a formal approach to higher dimensional relations, together with an example in 31 tone equal temperament using the formalism described earlier. We use the formalism of braids for our higher dimensional relations. We end with a section on musical applications of paths in graphs, generalising the chromatic scale.
\end{abstract}

Keywords: Scale Subsets, Similarity Composition, Harmonics

\section{Introduction}

Certain human processes relate to subsets. For example, a child may be given a naive set $A$ of cards featuring pictures of animals, and asked to return those featuring a picture of a cat. The collection of cards returned by the child forms a subset $B$ of the original set of cards. To give a musical example, a composer may write a piece, which is the unique constituent of a one element subset $B$ of the set $A$ of compositions that can be written with the available notation. Alternatively, our composer may define a larger subset $B$ of $A$ as their composition, from which a set of players will choose one element for a given performance.

Subsets of scales are commonly used to restrict musical possibilities. For example, the frequencies on an equally tempered piano form a subset of $\mathbb{R}$; the $\mathrm{C}$ major scale forms a subset of the set of frequencies on an equally tempered piano; the notes in a $\mathrm{C}$ major triad form a subset of the $\mathrm{C}$ major scale; the $\mathrm{Cs}$ form a subset of the notes in a $\mathrm{C}$ major triad.

Here we are interested in compositional techniques based on subsets of scales. We present a number of such techniques, built on theoretical notions, together with examples. We specify our interest to compositions that are designed to appeal to the memory, via similarity (for more on similarity in music, see [9]). Our methods using specified subsets are designed to be similar to techniques used in music that is already reasonably well-known.

Often technical considerations lead us to define a family of subsets of a given scale, from which we must choose one or more to make our music. This choice may be somewhat arbitrary. For example, consider piano music. It is standard to give the notes of an equally tempered piano the set of 
frequencies $\left\{2^{\frac{n}{12}} \alpha \mid-48 \leq n \leq 39\right\} \subset \mathbb{R}$, where $\alpha=$ 440. However the musical world would be little altered if we worked with a different subset of $\mathbb{R}$ by setting $\alpha=441$ instead.

From now on we write subset in sans-serif font to emphasise the role of such in our constructions.

There are music files to accompany this paper [15].

\section{Sounds}

Let us recall the formalism of chromatic combinations (see [14], Section 2).

Let $a, d, f \in \mathbb{R}_{+}$. Let $\chi_{I}$ denote the indicator function of an interval $I \subset \mathbb{R}$. For a function $\eta: \mathbb{R} \rightarrow \mathbb{R}$ of bounded support, we call the infimum of the support of $\eta$ the start of $\eta$, and we call the supremum of the support of $\eta$ the end of $\eta$. If $\eta, \eta^{\prime}: \mathbb{R} \rightarrow \mathbb{R}$ and the end of $\eta$ is equal to the start of $\eta^{\prime}$, we call $\eta+\eta^{\prime}$ the concatenation of $\eta$ and $\eta^{\prime}$. We define a pure tone of amplitude $a$, duration $d$, and frequency $f$, to be given by a function from $\mathbb{R}$ to $\mathbb{R}$ sending $t$ to $a \chi_{(o, o+d)} \sin (2 \pi f t+\phi)$, for some $o, \phi \in \mathbb{R}$.

Points on the stave are given by frequencies, with the A above middle $\mathrm{C}$ corresponding to $440 \mathrm{~Hz}$, and the operation of raising by a semitone corresponding to multiplication by $2^{\frac{1}{12}}$. Points on the stave are also given by integers, with middle $\mathrm{C}$ corresponding to 0 , and the operation of raising by a semitone corresponding to addition of 1 .

Fix $a \in \mathbb{R}_{+}$. We define a chromatic combination of duration $d$ to be given by a function from $\mathbb{R}$ to $\mathbb{R}$ sending $t$ to $a \chi_{(o, o+d)} \sum_{j=1}^{p} \sin \left(2 \pi f_{j} t+\phi_{j}\right)$, for some $o, \phi_{j} \in$ $\mathbb{R}$, and frequencies $f_{j}$ given by points on the stave, $j=$ $1, \ldots, p$. We sometimes abuse notation, and identify chromatic combinations with identical frequencies $f_{j}$, amplitude $a$ and duration $d$.

Note that the software we have used to turn our compositions into audio files manipulates our chromatic combinations somewhat.

We are interested in chromatic combinations because of their formal similarity to notes played on an equally tempered piano. This gives a similarity between music made with chromatic combinations and a body of existing music.

As well as sequences of chromatic combinations, we are also interested in overlapping sequences of notes given by decaying sawtooth waves. Let $a, d \in \mathbb{R}_{+}$. Let $o \in \mathbb{R}$. We denote by $\Psi_{(o, o+d)}: \mathbb{R} \rightarrow \mathbb{R}$ the function that is zero outside $(o, o+d)$, and is given by $\Psi_{(o, o+d)}(t)=a-$ $\frac{a}{d}(t-o)$ for $t \in(o, o+d)$. The function thus decays linearly from $a$ to 0 over the interval $(o, o+d)$. Given a sequence $\vec{f}=\left(f_{1}, f_{2}, \ldots, f_{n}\right)$ of frequencies $f_{i} \in \mathbb{R}$, we write $\Phi(\vec{f})=\Phi_{a, d}(\vec{f})=\sum_{i=1}^{n} \Psi_{\left((i-1) \frac{d}{2},(i+1) \frac{d}{2}\right)} \Sigma_{f_{i}}$, where $\Sigma_{f_{i}}$ denotes a sawtooth wave with frequency $f_{i}$. Thus $\Phi(\vec{f})$ is a function from $\mathbb{R}$ to $\mathbb{R}$ representing a sequence of sawtooth notes of duration $d$, whose frequencies are given by $\vec{f}$, played successively with an overlap of duration $\frac{d}{2}$.

We are interested in sawtooth waves because their harmonics are integer multiples of a fundamental, like notes played on a stringed instrument.

\section{Intersecting Accompaniments}

Suppose we are given a composition $A$ whose notes are given by points on the stave. We can form a subset $X$ of points on the stave which intersects the set of notes of that composition nontrivially, and accompany the composition with a sequence of notes taken from the set $X$ to form a composition $B$. There is then some agreement between the accompaniment and $A$. It may be desirable for $X$ to include notes that do not belong to $A$, to differentiate $B$ from $A$.

\section{Similarity Concatenation Compositions}

Suppose we are given a set of musical phrases that contains a phrase of a single note, is closed under concatenation, and is closed under the action of a set of similarity transformations, that send phrases to similar phrases; we can then form such a composition by starting with the note, applying a sequence of similarity transformations to obtain a sequence of notes, concatenating these to form a phrase, applying a sequence of similarity transformations to obtain a sequence of phrases, concatenating these to form a longer phrase, etc. [13]. We call a composition generated in this way a similarity concatenation composition.

We give some examples. Let $d \in \mathbb{R}_{+}$. Let $\mathcal{S}$ be a set of sounds of duration $d$, with a partial ordering. We call such a set a scale of notes of duration $d$.

Let $l$ be a natural number. Let $R=\mathbb{Z} / l \mathbb{Z}$. Let $\mathcal{S}_{l}$ denote the scale of notes of duration $(l-1) d$ whose elements correspond to elements $a=\left(a_{s}\right)_{s \in \mathcal{S}} \in R^{\mathcal{S}}$, and consist of the superposition of the sounds $\pi(a, s)$, where $\pi(a, s)$ is silence for $(l-1) d$ seconds if $a_{s}=0$, and silence for $\left(a_{s}-1\right) d$ seconds, followed by $s$, followed by silence for $\left(l-1-a_{s}\right) d$ seconds if $a_{s} \neq 0$. We order $R$ via its identification with the set $\{0,1,2, \ldots, l-1\}$. We order $\mathcal{S}_{l}$ lexicographically.

Suppose we are given $a, v_{1}, v_{2}, \ldots, v_{k} \in R^{\mathcal{S}}$. Consider the image of the map $R^{k} \rightarrow R^{\mathcal{S}}$ that sends $\alpha$ to $\alpha+a$. This determines a set of notes in $\mathcal{S}_{l}$ which we order via the lexicographic ordering on $R^{k}$, and concatenate to give a similarity concatenation composition of duration $l^{k}(l-1) d$.

We consider the case where the vector spaces $R^{k}$ and $R^{\mathcal{S}}$ above are permutation $R G$-modules, generated by transitive $G$-sets.

Let $G$ be a finite group, and $H$ a subgroup of $G$. Let $G / H$ be the set of left cosets of $H$ in $G$, and $R[G / H]$ the permutation $R G$-module generated by this set. Let $r=|G / H|$. Suppose we are given a map $c$ from $G / H$ to a scale $\mathcal{S}$. We inherit a map $\iota$ from $R[G / H]$ to $\mathcal{S}_{l}$

Suppose $K$ is a subgroup of $G$, and $x$ is a double coset in $K \backslash G / H$. The canonical isomorphism $\operatorname{Hom}_{G}(R[G / K],[G / H]) \cong R[K \backslash G / H]$ means that $x$ determines a $G$-homomorphism $\phi$ from $R[G / K]$ to $R[G / H]$. Composing $\phi$ with the map $\iota$ from $R[G / H]$ to $\mathcal{S}_{l}$, we obtain a $\operatorname{map} \tilde{\phi}$ from $R[G / K]$ to $\mathcal{S}_{l}$. 
Suppose we are given a linear ordering of the set $G / K$, and $|G / K|=n$. Such an ordering determines an identification of $R[G / K]$ with $R^{n}$. The lexicographic ordering on $R^{n}$ thus determines a linear ordering on $R[G / K]$, which restricts to the linear ordering of $G / K$. We have a similarity concatenation composition of duration $l^{n}(l-1) d$ corresponding to the images of elements of $R[G / K]$ under $\tilde{\phi}$, concatenated in this order.

A common compositional technique is to begin and end a piece in the same key; a key is a subset of the set of notes which are played during the piece. This technique of beginning and ending with the same subset can be applied in a similarity concatenation composition by using similarity transformations that compose to give the identity. A particularly strong form is the following:

Begin with a note, apply a sequence of similarity transformations which compose to the identity to obtain a sequence of notes, concatenate these to form a phrase, apply a sequence of similarity transformations which compose to the identity to obtain a sequence of phrases, concatenate these to form a longer phrase, etc.. We call a composition generated in this way a returning similarity concatenation composition. In such a composition, a special role is played by the one element subset of the set of notes, consisting of the single note with which the piece begins and ends. This single note is returned to repeatedly during the piece.

\section{Quasi-relations}

Selecting scales in $\mathbb{R}$ from which the frequencies of the notes of a composition are selected is a common practice (see [3], for example). Here we discuss using approximate relations between integers, under multiplication, to derive associated equally tempered scales in $\mathbb{R}$. These are subsets of $\mathbb{R}$ which come with associated endomorphisms, which come with precise relations between them.

Let $p$ be a prime, and $\mathcal{P}$ the set of primes $\leq p$. Let $\delta>0$ be a real number. We define a quasi-relation to be an expression $\rho=2^{a_{2}} 3^{a_{3}} \ldots p^{a_{p}}$, where $a_{i} \in \mathbb{Z}$ for $i \in \mathcal{P}$, and $1<\rho \leq 1+\delta$.

Suppose we are given quasi-relations $\rho_{j}=2^{a_{2 j}} 3^{a_{3 j}} \ldots p^{a_{p j}}$, where $1 \leq j \leq|\mathcal{P}|-1$. Suppose the vectors $\left(a_{2 j}, \ldots, a_{p j}\right)$ are linearly independent, for $1 \leq j \leq|\mathcal{P}|-1$. Solving the set of $|\mathcal{P}|-1$ equations in $|\mathcal{P}|$ variables,

$$
a_{2 j} x_{2}+a_{3 j} x_{3}+\ldots+a_{p j} x_{p}=0,1 \leq j \leq|\mathcal{P}|-1,
$$

yields a family of solutions $\left(x_{2}, x_{3}, \ldots, x_{p}\right)=\lambda y$, where $\lambda$ runs through elements of $\mathbb{Q}$, and $y \in \mathbb{Q}^{\mathcal{P}}$ is fixed. There consequently exist integral solutions, of which we fix one $z=\left(z_{2}, z_{3}, \ldots, z_{p}\right)$.

Let us assume $z_{2}>0$ and fix $c \in \mathbb{R}$. We take an equally tempered scale $\mathfrak{E}$, with frequencies given by $c .2^{\frac{n}{z_{2}}}$, as $n$ runs through the elements of $\mathbb{Z}$. If we call the interval given by the frequency ratio $2^{\frac{1}{z_{2}}}$ a basic interval, this scale has $z_{2}$ basic intervals to an octave. For $q \in \mathcal{P}$, this scale has an endomorphism $e_{q}$, given by multiplication by $2^{\frac{z_{q}}{z_{2}}}$, which we think of as an approximation to multiplication by $q$. In place of the quasi-relations $\rho_{j}$ we have the relations $r_{j}=1$, where $r_{j}=e_{2}^{a_{2 j}} e_{3}^{a_{3 j}} \ldots e_{p}^{a_{p j}}$, for $1 \leq j \leq|\mathcal{P}|-1$.

For example, let us take $p=5$ and $\delta=\frac{1}{40}$. Consider the quasi-relations $2^{-4} \cdot 3^{4} \cdot 5^{-1}$ and $2^{7} \cdot 5^{-3}$. In $\mathbb{Z}^{\{2,3,5\}}$ we have associated linearly independent vectors $(-4,4,-1)$ and $(7,0,-3)$. The equations

$$
\begin{array}{r}
-4 x_{2}+4 x_{3}-x_{5}=0 \\
7 x_{2}-3 x_{5}=0
\end{array}
$$

have an integral solution $(12,19,28)$. If we take $c=440$ we recover the equally tempered scale $\mathfrak{E}_{12}$ commonly used on a piano, with 12 basic intervals to an octave.

For another example, let us take $p=7$, and $\delta=$ $\frac{1}{80}$. Consider the quasi-relations $2^{-4} .3^{4} .5^{-1}, 2.3^{2} .5^{-3} .7$, and $2^{-5} \cdot 3^{-1} \cdot 5^{-2} \cdot 7^{4}$. In $\mathbb{Z}^{\{2,3,5,7\}}$ we have associated linearly independent vectors $(-4,4,-1,0),(1,2,-3,1)$, and
$(-5,-1,-2,4)$. Applying row reduction to the equations

$$
\begin{aligned}
-4 x_{2}+4 x_{3}-x_{5} & =0, \\
x_{2}+2 x_{3}-3 x_{5}+x_{7} & =0, \\
-5 x_{2}-x_{3}-2 x_{5}+4 x_{7} & =0,
\end{aligned}
$$

we obtain an integral solution $(31,49,72,87)$. Taking $z$ to be this element, and $c=440$, we obtain an equally tempered scale $\mathfrak{E}_{31}$ with 31 basic intervals to an octave, cf. [11].

Let us remark that many relations between the endomorphisms $e_{q}$ can be exposed, by multiplying the elements $r_{j}$ together.

\section{Connected Triads and Triheptads}

Consider the equally tempered scale with 12 basic intervals to the octave, whose frequencies are given by the product of 440 and a power of $2^{\frac{1}{12}}$. We identify this scale with $\mathbb{Z}$ so that 0 corresponds to middle $\mathrm{C}, e_{2}$ corresponds to addition of $12, e_{3}$ corresponds to addition of 19 , and $e_{5}$ corresponds to addition of 28 . It is common practice to identify various subsets of our scale $\mathbb{Z}$

Consider subsets of $\mathbb{Z}$ of the form $\left\{e_{2}^{n}(\alpha) \mid n \in \mathbb{Z}\right\}$ for $\alpha \in \mathbb{Z}$; these are the elements of $\mathbb{Z} / 12 \mathbb{Z}$. Consider the subset of $\mathbb{Z}$ given by

$$
\left\{e_{3}^{m} e_{2}^{n}(\alpha) \mid m, n \in \mathbb{Z}, 0 \leq m \leq 6\right\},
$$

for $\alpha \in \mathbb{Z}$; such a subset is the major scale corresponding to $e_{3}(\alpha)$. Consider the subset of the major scale corresponding 
to $e_{3}(\alpha)$ given by

$$
\left\{\eta e_{2}^{n}(\alpha) \mid n \in \mathbb{Z}, \eta \in\left\{1, e_{3}, e_{5}\right\}\right\} .
$$

This is the subdominant of the major scale.

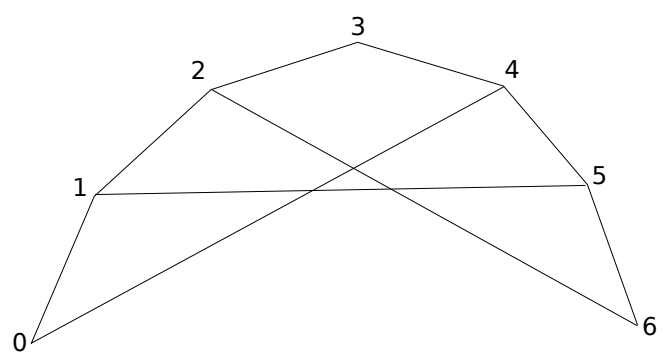

Figure 1. The graph $\Lambda$.

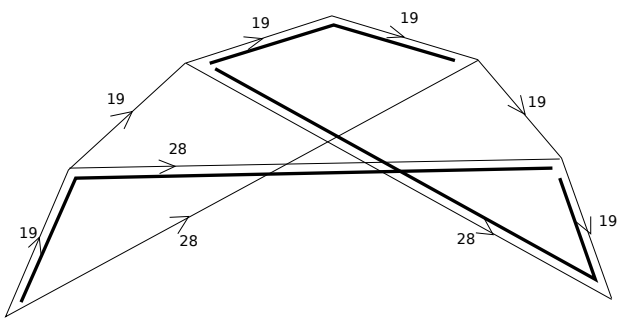

Figure 2. The scale $\Lambda_{12}$ and the connected triheptad $\{0,1,5\},\{2,5,6\},\{2,3,4\}$.

Consider the subset of the major scale corresponding to $e_{3}(\alpha)$ given by

$$
\left\{\eta e_{3} e_{2}^{n}(\alpha) \mid n \in \mathbb{Z}, \eta \in\left\{1, e_{3}, e_{5}\right\}\right\} .
$$

This is the tonic of the major scale. Consider the subset of the major scale corresponding to $e_{3}(\alpha)$ given by

$$
\left\{\eta e_{3}^{2} e_{2}^{n}(\alpha) \mid n \in \mathbb{Z}, \eta \in\left\{1, e_{3}, e_{5}\right\}\right\} .
$$

This is the dominant of the major scale. The major scale is the union of the subdominant, the tonic, and the dominant. The intersection of the subdominant and the tonic is the element of $\mathbb{Z} / 12 \mathbb{Z}$ indexed by $e_{3}(\alpha)$. The intersection of the tonic and the dominant is the element of $\mathbb{Z} / 12 \mathbb{Z}$ indexed by $e_{3}^{2}(\alpha)$. The intersection of the subdominant and the dominant is empty.

We abstract this situation as follows. Let $\Lambda$ denote the graph depicted in Figure 1. We define a connected triad in $\Lambda$ to be a connected subgraph of $\Lambda$ with three vertices. We define a connected triheptad in $\Lambda$ to be a sequence $\left(L_{1}, L_{2}, L_{3}\right)$, where $L_{i}$ is a connected triad in $\Lambda$ for $i=1,2,3$, where $\left|L_{1} \cap L_{2}\right|=\left|L_{2} \cap L_{3}\right|=1$, and where $\left|L_{1} \cap L_{3}\right|=0$. Every vertex of $\Lambda$ is a vertex of some $L_{i}$, if $\left(L_{1}, L_{2}, L_{3}\right)$ is a connected triheptad.

This relates to our subsets of $\mathbb{Z}$, since $\Lambda$ can be identified with the graph whose vertices are elements of $\mathbb{Z} / 12 \mathbb{Z}$ contained in a given major scale, and whose edges connect vertices $v, w$ when $w=e_{i}(v), i=3,5$. We have a connected triheptad given by the subdominant, followed by the tonic, followed by the dominant. However, there are many other connected triheptads, for example that depicted in Figure 2.

Subdominant, tonic and dominant chords are commonly used in sequences to structure simple pieces of music. The internal associations in a connected triheptad $\left(L_{1}, L_{2}, L_{3}\right)$ mean that such can play a similar role, in case the graph $\Lambda$ arises harmonically. We give two examples.

Example 6.1. In this example, the $j^{\text {th }}$ bar contains scale elements corresponding to elements of $L_{i(j)}$, where $i(j)=j$ $\bmod 3$. We take as $\Lambda_{12}$ the $\mathrm{C}$ major scale. We take $L_{1}=$ $\{0,1,5\}, L_{2}=\{2,5,6\}, L_{3}=\{2,3,4\}$. Our composition consists of two piano lines, one in the range from the note an octave below middle $\mathrm{C}$ to the $\mathrm{B}$ below middle $\mathrm{C}$, and the other in the range from middle $\mathrm{C}$ to the note two octaves above middle C. For $j \leq 10$ the $j^{\text {th }}$ bar of the bass line consists of the triad $L_{i(j)}$ repeated four times as an ascending triplet of duration $\frac{1}{2}$, with an exception in the $10^{\text {th }}$ bar where the line is brought to close with the note with which it began. The upper line meanders within its range, with all notes in the $j^{\text {th }}$ bar taken from the triad $L_{i(j)}$; we have included some repetition of rhythmic motifs.

Example 6.2. Let $\alpha=-10$. Consider subsets of $\mathbb{Z}$ of the form $\left\{e_{3}^{n}(\alpha) \mid n \in \mathbb{Z}\right\}$ for $\alpha \in \mathbb{Z}$; these are the elements of $\mathbb{Z} / 19 \mathbb{Z}$. Consider the subset of $\mathbb{Z}$ given by $\tilde{\Lambda}_{19}=\left\{e_{2}^{m} e_{3}^{n}(\alpha) \mid m, n \in \mathbb{Z}, 0 \leq m \leq 6\right\}$ for $\alpha \in \mathbb{Z}$. The elements $\Lambda_{19}$ of $\mathbb{Z} / 19 \mathbb{Z}$ contained in $\tilde{\Lambda}_{19}$ can be identified with the vertices of $\Lambda$ where edges connect vertices $v, w$ when $w=e_{i}(v), i=2,5$. We take $L_{1}=\{0,1,4\}, L_{2}=\{1,2,5\}$, $L_{3}=\{2,3,6\}$.

Our composition has a piano part, which is a similarity concatenation composition whose 5 scale elements consist of 4 bar chord sequences, together with an intersecting accompaniment of pure tones, each of 8 bars duration. The duration of each bar is $\frac{15}{4}$. The chord sequences have three chords, taken from $\tilde{L}_{1}, \tilde{L}_{2}, \tilde{L}_{3}$ respectively, where $\tilde{L}_{i}$ denotes the union of the equivalence classes in $\mathbb{Z} / 19 \mathbb{Z}$ given by elements of $L_{i}$.

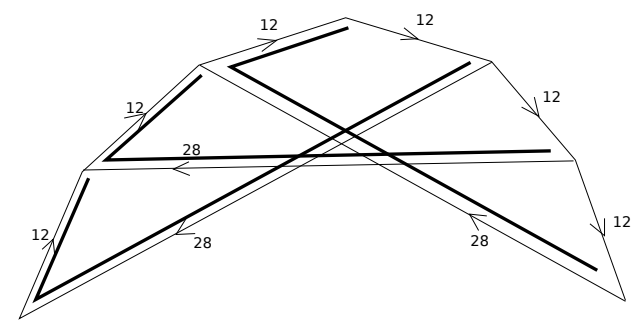

Figure 3. The scale $\Lambda_{19}$ and the connected triheptad $\{0,1,4\},\{1,2,5\},\{2,3,6\}$.

Let

$$
\begin{aligned}
& S_{1}=\left\{e_{5}^{-1} e_{3}^{4}(\alpha)\right\}, \\
& S_{2}=\left\{e_{3}^{3}(\alpha), e_{5}^{-1} e_{3}^{3}(\alpha)\right\}, \\
& S_{3}=\left\{e_{3}^{2}(\alpha), e_{2} e_{3}^{2}(\alpha), e_{5}^{-1} e_{3}^{2}(\alpha)\right\}, \\
& S_{4}=\left\{e_{3}(\alpha), e_{2} e_{3}(\alpha), e_{5}^{-1} e_{3}(\alpha)\right\}, \\
& S_{5}=\left\{\alpha, e_{2}(\alpha)\right\} .
\end{aligned}
$$

For $i=1,2,3,4,5$, let $v_{i}$ denote the scale element 
consisting of the chord $S_{i}$ played for one bar, followed by the chord $e_{3}^{-1} e_{2}\left(S_{i}\right)$ played for one bar, followed by the chord $e_{3}^{-2} e_{2}^{2}\left(S_{i}\right)$, played for two bars. We set $\mathcal{S}=$ $\left\{v_{1}, v_{2}, v_{3}, v_{4}, v_{5}\right\}$. Our similarity concatenation composition is given by the scale $\mathbb{Z} / 2 \mathbb{Z}[\mathcal{S}]$, ordered lexicographically and concatenated.

We take an intersecting accompaniment consisting of an ascending line of pure tones alternated with a descending line of pure tones. Each pure tone is eight bars long. The ascending line is given by the sequence $-16,-13,-11,-9,-6,-4,1,3$. The descending line is given by the sequence $17,14,12,9,7,5,2,0$. The notes in the ascending line are chosen not to belong to $\tilde{L}_{1}, \tilde{L}_{2}$, or $\tilde{L}_{3}$. All but the first note of the descending line belong to $\tilde{L}_{1}, \tilde{L}_{2}$, or $\tilde{L}_{3}$. The first three notes and the last three notes of the descending line differ by an octave.

\section{A Cubist Example}

In this section we construct an example using cubist techniques $[4,6]$, generalising previously described methods ([14], Section 4).

Take a strictly increasing map $\iota: \mathbb{Z} \rightarrow \mathbb{Z}$. This map is given equivalently by a subset $\Omega \subseteq \mathbb{Z}$ that is unbounded from above and below, with an element $\omega \in \Omega$. Indeed, such data emerges when we write $\Omega=\iota(\mathbb{Z})$ and $\omega=\iota(0)$.

We recall the formalism of cubist sets [6]. For $r$ a natural number, let $\underline{r}=\{1, \ldots, r\}$.

Let $E=\mathbb{R}^{\oplus \underline{r}}$ denote Euclidean space, of dimension $r$. Let $\epsilon_{i}$ denote the standard basis element of $E$, for $i \in \underline{r}$.

Suppose $\Sigma \subset \underline{r}$. Let $F_{\Sigma}=F_{1} \times \ldots \times F_{r} \subset E$, where $F_{i}=[0,1]$, if $i \in \Sigma$, and $F_{i}=\{0\}$, if $i \notin \Sigma$.

Let $\mathcal{Z}=\mathcal{Z}_{r}$ denote the polytopal complex, homeomorphic to $E$, whose $i$-dimensional cells are $i$-cubes in $E$ of the form $x+F_{\Sigma}, x \in \mathbb{Z}^{r},|\Sigma|=i$.

Let $H=(1,1, . ., 1)^{\perp} \subset \mathbb{R}^{r}$. Let $p: E \rightarrow H$ denote orthogonal projection.

We say a polytopal subcomplex $\mathcal{C} \subset \mathcal{Z}$ is cubist if the projection $p: \mathcal{C} \rightarrow H$ is a homeomorphism.

If $\mathcal{C}$ is a cubist complex, we write $\mathcal{X}=\mathcal{C} \cap \mathbb{Z}^{r}$, and call $\mathcal{X}$ a cubist set in $\mathbb{Z}^{r}$. We denote by $s_{\mathcal{X}}$ the map from $\mathbb{Z}^{r}$ to $\mathbb{Z}$ given by $s_{\mathcal{X}}(x+(k, k, \ldots, k))=k$ for $x \in \mathcal{X}, k \in \mathbb{Z}$.

Let $\mathcal{X}_{1}, \ldots, \mathcal{X}_{m} \subset \mathbb{Z}^{r}$ be cubist subsets. Let $s_{1}=$ $s_{\mathcal{X}_{1}}, \ldots, s_{m}=s_{\mathcal{X}_{m}}$. These maps define our scale $\mathfrak{S}$ indexed by $\mathbb{Z}^{r} \times D$, where $(x, d) \in \mathbb{Z}^{r} \times D$ corresponds to a chromatic combination of duration $d$ whose partials are given by positions $\iota\left(s_{1}(x)\right), \ldots, \iota\left(s_{m}(x)\right)$ on the stave. Such scales generalise the scales of chromatic combinations indexed by $\mathbb{Z}$ which arise in case $r=1$ and $\iota=1_{\mathbb{Z}}$, and may be played on certain synthesisers.

Translation by $z \in \mathbb{Z}^{r}$ defines a transformation of our scale, as in the $r=1$ case. For $z \in \mathbb{Z}^{r}$ we define $\tau_{z}$ to be translation by $z$.

For $z \in \mathbb{Z}^{r}$ and $n$ a positive integer, let $\Delta_{z, n}$ denote the transformation of $\mathbb{Z}^{r}$ given by dilation by $n$, centred at $z$. Thus $\Delta_{z, n}(x)=n(x-z)+z$, for $x \in \mathbb{Z}^{r}$.
For $z \in \mathbb{Z}^{r}$ and $1 \leq i \leq r$, let $r_{z, i}$ denote the transformation of $\mathbb{Z}^{r}$ given by reflection in the hyperplane through $z$, perpendicular to $\epsilon_{i}$.

We denote by $\mathcal{T}$ the set of transformations of $\mathbb{Z}^{r}$ consisting of transformations $\tau_{z}, \Delta_{z, n}$, and $r_{z, i}$. We denote by $\mathcal{E}_{\mathbb{Z}^{r}}$ the monoid of transformations of $\mathbb{Z}^{r}$ generated by $\mathcal{T}$. We thus have an action of $\mathcal{E}_{\mathbb{Z}^{r}}$ on $\mathbb{Z}^{r}$.

We write $\mathcal{E}_{D}=\mathbb{R}_{+}$. Thus $\mathcal{E}_{D}$ acts on $D$ by multiplication.

We describe similarity concatenation compositions with scale $\mathfrak{S}$.

Let $w$ be a natural number, and let $\lambda_{1}, \ldots, \lambda_{w}$ be natural numbers. For $i=1, \ldots, w$ we take maps $t_{i}: \lambda_{i} \rightarrow \mathcal{T} \times \mathcal{E}_{D}$. We insist that $t_{i}(1)$ is equal to the identity, for $i=1, \ldots, w$, but that $t_{i}(\xi)$ is different from the identity, for $\xi=2, \ldots, \lambda_{i}$ and $i=1, \ldots, w$.

For $i=1, \ldots, w$ we define maps $u_{i}: \underline{\lambda_{i}} \rightarrow \mathcal{E}_{\mathbb{Z}^{r}} \times \mathcal{E}_{D}$, by

$$
u_{i}(\xi)=t_{i}(\xi) t_{i}(\xi-1) \ldots t_{i}(1) .
$$

Let us fix an element $q=(x, d) \in \mathbb{Z}^{r} \times D$. Our composition is obtained by concatenating scale elements corresponding to elements $\left(\xi_{1}, \ldots, \xi_{w}\right)$ of $\underline{\lambda_{1}} \times \underline{\lambda_{2}} \times \ldots \times \underline{\lambda_{w}}$, ordered lexicographically. The element of $\overline{\mathfrak{S}}$ corresponding to $\left(\xi_{1}, \ldots, \xi_{w}\right)$ is given by $u_{1}\left(\xi_{1}\right) u_{2}\left(\xi_{2}\right) \ldots u_{w}\left(\xi_{w}\right) q$.

Example 7.1. We describe a returning similarity composition. Let $\omega$ be given by middle $\mathrm{C}$. Let $\Omega=$ $\{0,2,3,4,5,7,8,9,10,11\}+12 \mathbb{Z}$. We take

$$
\mathcal{X}_{1}=0 \times \mathbb{Z}
$$

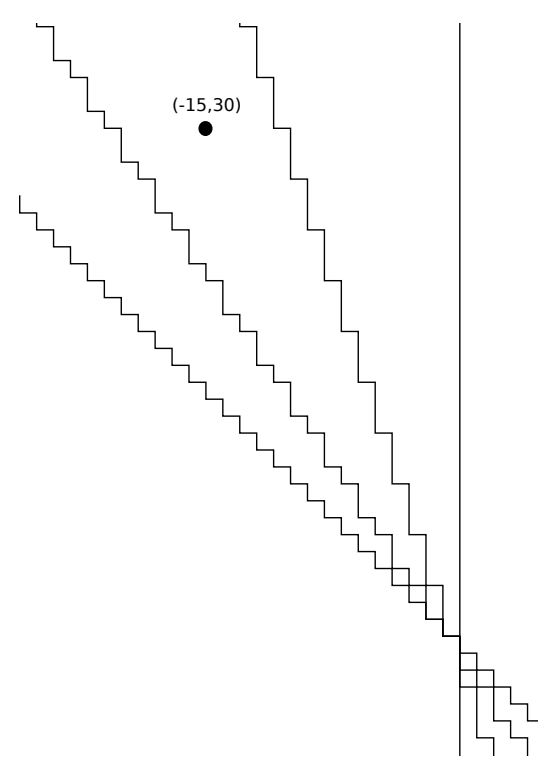

Figure 4. The cubist sets and first note of Example 7.1.

$$
\begin{aligned}
& \mathcal{X}_{2}=\{(0,0),(-1,0),(-1,1),(-1,2)\}+\mathbb{Z}(-1,3), \\
& \mathcal{X}_{3}=\{(0,0),(-1,0),(-1,1),(-2,1),(-2,2)\}+\mathbb{Z}(-2,3), \\
& \mathcal{X}_{4}=\{(0,0),(-1,0)\}+\mathbb{Z}(-1,1) .
\end{aligned}
$$

We take $q=\left((-15,30), \frac{2}{35}\right), w=4$. 
We take $\lambda_{4}=5$ and

$$
\begin{aligned}
& t_{4}(2)=\left(\tau_{(-1,0)}, 1\right), t_{4}(3)=\left(\tau_{(0,1)}, 1\right), \\
& t_{4}(4)=\left(\tau_{(1,0)}, 1\right), t_{4}(5)=\left(\tau_{(0,-1)}, 1\right) .
\end{aligned}
$$

We take $\lambda_{3}=7$ and

$$
\begin{aligned}
& t_{3}(2)=t_{3}(3)=t_{3}(4)=\left(\Delta_{(-15,30), 2}, 1\right), \\
& t_{3}(5)=\left(\Delta_{(-15,30), \frac{3}{4}}, 1\right), \\
& t_{3}(6)=\left(\Delta_{(-15,30), \frac{1}{2}}, 1\right), t_{3}(7)=\left(\Delta_{(-15,30), \frac{1}{3}}, 1\right) .
\end{aligned}
$$

We take $\lambda_{2}=5$ and

$$
\begin{aligned}
& t_{2}(2)=\left(r_{(-15,30), 2}, 2^{\frac{1}{4}}\right), t_{2}(3)=\left(r_{(-15,30), 1}, 2^{\frac{1}{4}}\right), \\
& t_{2}(4)=\left(r_{(-15,30), 2}, 2^{-\frac{1}{4}}\right), t_{2}(5)=\left(r_{(-15,30), 1}, 2^{-\frac{1}{4}}\right) .
\end{aligned}
$$

We take $\lambda_{1}=5$ and

$$
\begin{aligned}
& t_{1}(2)=\left(\tau_{(0,16)}, 2^{\frac{1}{4}}\right), t_{1}(3)=\left(\tau_{(16,0)}, 2^{\frac{1}{4}}\right), \\
& t_{1}(4)=\left(\tau_{(0,-16)}, 2^{-\frac{1}{4}}\right), t_{1}(5)=\left(\tau_{(-16,0)}, 2^{-\frac{1}{4}}\right) .
\end{aligned}
$$

We take an intersecting accompaniment that is given by an ascending scale with frequencies given by successive increasing elements of $\iota(\mathbb{Z})$, beginning with $\iota(14)$. Each note of the ascending scale accompanies a sequence of $\lambda_{3} \times \lambda_{4}=35$ scale elements.

\section{Concatenating Similarity Concatenation Compositions via Similarity}

We next move up a conceptual level, from the world of similarity concatenation compositions to the world of concatenations of similarity concatenation compositions. Passing to a higher conceptual level is a technique which has found striking applications in algebra [5, 12].

Suppose $\Omega$ denotes a finite set. Let

$c: \Omega \rightarrow\{$ finite disjoint unions of open intervals in $(0, d)\}$,

for some $d \in \mathbb{R}_{+}$. Given a map $f: \Omega \rightarrow \mathbb{R}$, we have an associated function $c(f): \mathbb{R} \rightarrow \mathbb{R}$ that sends $t$ to $\sum_{\omega \in \Omega} \sin (2 \pi f(\omega) t) \chi_{c(\omega)}$.

If we are given a sequence of functions $c$ and a sequence of maps $f$, say $c_{1}, \ldots, c_{m}$ and $f_{1}, \ldots, f_{n}$, we can concatenate the functions

$$
c_{1}\left(f_{1}\right), \ldots, c_{1}\left(f_{n}\right), c_{2}\left(f_{1}\right), \ldots, c_{2}\left(f_{n}\right), \ldots, c_{m}\left(f_{1}\right), \ldots, c_{m}\left(f_{n}\right)
$$

to obtain a composition. If the compositions $c_{i}\left(f_{j}\right)$ are similarity concatenation compositions, then this as a sequence of similarity concatenation compositions, concatenated via similarity. We can think of the subsets $f_{i}(\Omega)$ of $\mathbb{R}$ as the keys of our composition, which are run through in sequence $m$ times during the course of the piece.
Example 8.1. In our example $m=3$ and $n=4$, whilst $\Omega=$ $\left\{\omega_{0}, \omega_{1}, \omega_{2}, \ldots, \omega_{6}\right\}$ is a seven element set. The composition thus has three parts, each involving four choices of seven frequencies.

For $i=1,2,3,4$, the maps $f_{i}$ correspond to maps $\hat{f}_{i}$ from $\Omega$ to the set of points on the stave, which we identify with $\mathbb{Z}$. These maps all send $\omega_{0}$ to -24 , whilst

$$
\begin{aligned}
& \hat{f}_{1}\left(\omega_{1}\right)=\hat{f}_{2}\left(\omega_{1}\right)=\hat{f}_{3}\left(\omega_{1}\right)=13-24, \\
& \hat{f}_{1}\left(\omega_{2}\right)=\hat{f}_{2}\left(\omega_{2}\right)=\hat{f}_{4}\left(\omega_{1}\right)=20-24, \\
& \hat{f}_{1}\left(\omega_{3}\right)=\hat{f}_{3}\left(\omega_{2}\right)=\hat{f}_{4}\left(\omega_{2}\right)=25-24, \\
& \hat{f}_{2}\left(\omega_{3}\right)=\hat{f}_{3}\left(\omega_{3}\right)=\hat{f}_{4}\left(\omega_{3}\right)=29-24, \\
& \hat{f}_{i}\left(\omega_{4}\right)=\hat{f}_{i}\left(\omega_{1}\right)+\hat{f}_{i}\left(\omega_{2}\right)+24, \\
& \hat{f}_{i}\left(\omega_{5}\right)=\hat{f}_{i}\left(\omega_{1}\right)+\hat{f}_{i}\left(\omega_{3}\right)+24, \\
& \hat{f}_{i}\left(\omega_{6}\right)=\hat{f}_{i}\left(\omega_{2}\right)+\hat{f}_{i}\left(\omega_{3}\right)+24,
\end{aligned}
$$

for $i=1,2,3,4$.

For the first part $c_{1}$ of our composition we specify a scale of 8 elements, corresponding to the $2^{3}$ elements of $\left\{\omega_{1}, \omega_{4}\right\} \times$ $\left\{\omega_{2}, \omega_{5}\right\} \times\left\{\omega_{3}, \omega_{6}\right\}$; an element $\left(\alpha_{1}, \alpha_{2}, \alpha_{3}\right)$ corresponds to $\alpha_{1}$ followed by $\alpha_{2}$ followed by $\alpha_{3}$, each played with duration $\frac{1}{6}$. The function $c_{1}$ corresponds to these scale elements played in colexicographic order, followed by $\omega_{0}$ played with duration

2 . Here we assume $\omega_{1}<\omega_{2}, \omega_{3}<\omega_{4}$, and $\omega_{5}<\omega_{6}$.

For the second part $c_{2}$ of our composition, we use the formalism of similarity concatenation compositions derived from finite groups in section 4 . Let $l=3$, let $G=$ $\operatorname{Sym}\{1,2,3,4\}$, and let $H=\operatorname{Sym}\{1,2\} \times \operatorname{Sym}\{3,4\}$. Let $K$ denote the subgroup of $G$ generated by $H$ and the permutation (1 3) $(24)$. Then $K$ is isomorphic to $D_{8}$. Let $x$ denote the double coset $K(23) H$.

We identify $G / H$ with the set of 2 -element subsets of $\{1,2,3,4\}$, which we in turn identify with $\Omega \backslash\left\{\omega_{0}\right\}$ via

$$
\begin{aligned}
\{1,2\} \mapsto \omega_{1}, \quad\{1,3\} \mapsto \omega_{2}, \quad\{1,4\} \mapsto \omega_{3}, \\
\{2,3\} \mapsto \omega_{4}, \quad\{2,4\} \mapsto \omega_{5}, \quad\{3,4\} \mapsto \omega_{6} .
\end{aligned}
$$

We order the left cosets of $K$ in $G$ as $K<\left(\begin{array}{lll}1 & 3 & 2\end{array}\right) K<$ (1 2 2 3$) K$.

For $i=1,2,3,4$, the section $c_{2}\left(f_{i}\right)$ is given by the similarity concatenation composition associated with this data, via the map $\hat{f}_{i}$, with $d=\frac{1}{4}$, followed by $\omega_{0}$ played with duration $\frac{1}{2}$, then silence of duration $\frac{3}{2}$.

For the third part $c_{3}$ of our composition, we use the formalism and notation of similarity concatenation compositions of section 4 . Let $a$ denote the sequence consisting of silence, followed by $\omega_{1}$, followed by silence, followed by $\omega_{2}$, followed by silence, followed by $\omega_{2}$, followed by silence, followed by $\omega_{3}$, followed by silence, followed by $\omega_{3}$, followed by silence, followed by $\omega_{1}$, where each element of this 12 term sequence has duration $\frac{1}{8}$. Let $v_{1}$ denote the sequence consisting of a chord of $\omega_{0}, \omega_{1}, \omega_{2}, \omega_{3}$ followed by three silences, played three times in succession, where each element of this 12 term sequence has duration $\frac{1}{8}$. Let $v_{2}$ denote 
the sequence consisting of two silences followed by a chord of $\omega_{1}, \omega_{2}, \omega_{4}$, followed by three silences followed by a chord of $\omega_{2}, \omega_{3}, \omega_{6}$ followed by three silences, followed by a chord of $\omega_{1}, \omega_{3}, \omega_{5}$ followed by a silence, where each element of this 12 term sequence has duration $\frac{1}{8}$.

Let $l=2$ and $\mathcal{S}=\left\{a, v_{1}, v_{2}\right\}$, identified as a set of coordinate vectors in $(\mathbb{Z} / 2 \mathbb{Z})^{\mathcal{S}}$. For $i=1,2,3,4$, the section $c_{3}\left(f_{i}\right)$ is given by the similarity concatenation composition associated with this data, via the map $\hat{f}_{i}$.

\section{Higher Dimensional Relations}

One technique for constructing a musical piece is to begin with a note, then take a sequence of successive notes that are close to form a phrase, then construct a successive sequence of phrases that are close to form a 2-phrase, then construct a successive sequence of 2-phrases that are close to form a 3phrase, etc. (cf. [1])

Here we give a formalism for such a procedure, and apply it to form a composition in 31 tone equal temperament $\mathfrak{E}_{31}$, using the relations we derived the scale from. In the process we identify a subset of the 31 tones which we draw our notes from.

Let $S$ denote a set. We define a 0-path to be an element of $S$. We define the source and target of a 0 -path to be $\circ$. For $n \geq 1$ we define an $n$-path $P$ to be a sequence $\left(a_{0}, a_{1}, \ldots, a_{n}\right)$ of $n-1$-paths with the same source and target. The source of $P$ is $a_{n}$ and the target of $P$ is $a_{0}$.

The length of $P$ is $l(P)=n$. If $P$ and $Q$ are $n$-paths, such that the target of $P$ is equal to the source of $Q$, we denote by $Q P$ the concatenation of $P$ and $Q$. We thus have $l(Q P)=l(P)+l(Q)$.

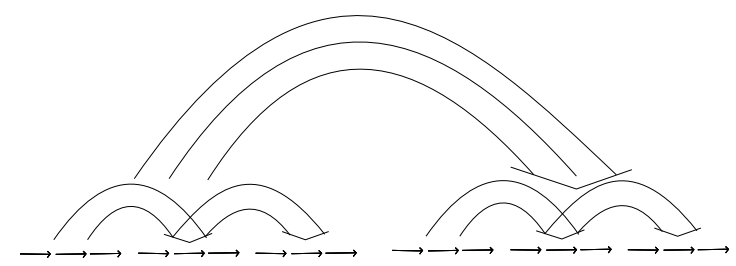

Figure 5. Relation between relations between relations.
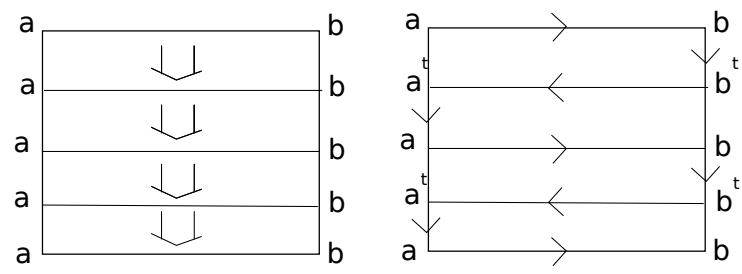

Figure 6. Sequence of scale elements $D(P)$ from a 2-path $P$.

We define an $n$-arrow $a$ to be a an $n$-path $\left(a_{0}, a_{1}\right)$ of length 1. We define the degree of $a$ to be $d(a)=l\left(a_{0}\right)+l\left(a_{1}\right)$. Suppose $a_{0}$ and $a_{1}$ are $n-1$-paths both of which have source and target $s$ and $t$ respectively, and $c$ denotes the $n$-arrow $\left(a_{0}, a_{1}\right)$. If $\alpha$ is an $n-1$-path with target $s$, and if $\beta$ is an $n-1$ path with source $t$, then we call $\left(\beta a_{0} \alpha, \beta a_{1} \alpha\right)$ the idextension of $c$ by $\alpha$ and $\beta$. We call such an $n$-arrow an idextension of $c$.

We derive sequences of elements of $S$ from our $n$-paths as follows: Let $t$ denote an endomorphism of $S$. Let $x^{r}$ denotes the reverse of a sequence $x$ in $S$. For $n=1$, and $P$ an $n$-path, let $D(P)=P$. For $n \geq 2$, given an $n$-path $P=\left(a_{0}, \ldots, a_{n}\right)$, let $D(P)$ denote the concatenation of $D\left(a_{0}\right), D\left(a_{1}\right)^{r . t}, D\left(a_{2}\right)$, $D\left(a_{3}\right)^{r . t}, \ldots$,

The elements of $S$ featuring in $D\left(a_{0}\right), D\left(a_{1}\right)^{r . t}$ etc. form a subset of the set of elements of $S$ featuring in $D(P)$.

Example 9.1. Our compositional strategy is to successively pick, for $2 \leq n \leq m$, for some $m$, a set of primitive $n$ arrows of low degree, which are generated by primitive $n-1$ arrows. Here we say an arrow is generated by primitive $n-1$ arrows if its source and target are concatenated idextensions of primitive $n-1$-arrows. The primitive 1 -arrows are given by the endomorphisms $e_{q}$ of our scale.

Let $S=\mathfrak{E}_{31}$, an equally tempered 31 tone scale. Let $t=e_{2}$ be given by multiplication by 2 .

As primitive 1-arrows we take $\left(e_{q}(s), s\right)$, for $s \in S$ and $q \in\{2,3,5,7\}$.

Let $o_{0}=440.2^{-100 / 31}$. We define

$$
\begin{aligned}
P_{1}= & \left(o_{1}, e_{3}^{-1} e_{2}^{3}\left(o_{0}\right), e_{2}^{3}\left(o_{0}\right), e_{2}^{2}\left(o_{0}\right), e_{2}\left(o_{0}\right), o_{0}\right) \\
& P_{1}^{\prime}\left(o_{1}, e_{5}^{-1} e_{3}^{2}\left(o_{0}\right), e_{3}^{2}\left(o_{0}\right), e_{3}\left(o_{0}\right), o_{0}\right)
\end{aligned}
$$

where $o_{1}=e_{3}^{-2} e_{2}^{3}\left(o_{0}\right)=e_{2}^{-1} e_{5}^{-1} e_{3}^{2}\left(o_{0}\right)$. We define $R_{1}=$ $\left(P_{1}^{\prime}, P_{1}\right)$.

We define

$$
\begin{aligned}
& P_{2}=\left(o_{2}, e_{3} e_{2}\left(o_{1}\right), e_{2}\left(o_{1}\right), o_{1}\right) \\
& P_{2}^{\prime}=\left(o_{2}, e_{5} e_{3}^{-1} e_{5}\left(o_{1}\right), e_{3}^{-1} e_{5}\left(o_{1}\right), e_{5}\left(o_{1}\right), o_{1}\right)
\end{aligned}
$$

where $o_{2}=e_{5}^{-1} e_{3} e_{2}\left(o_{1}\right)=e_{7}^{-1} e_{5} e_{3}^{-1} e_{5}\left(o_{1}\right)$. We define $R_{2}=\left(P_{2}^{\prime}, P_{2}\right)$.

We define

$$
\begin{aligned}
P_{3}= & \left(o_{3}, e_{5} e_{7}^{-1} e_{2}^{3}\left(o_{2}\right), e_{7}^{-1} e_{2}^{3}\left(o_{2}\right), e_{2}^{3}\left(o_{2}\right), e_{2}^{2}\left(o_{2}\right), e_{2}\left(o_{2}\right), o_{2}\right) \\
P_{3}^{\prime}= & \left(o_{3}, e_{5}^{-1} e_{7} e_{2}^{-1} e_{3}^{-1} e_{7}\left(o_{2}\right), e_{7} e_{2}^{-1} e_{3}^{-1} e_{7}\left(o_{2}\right)\right. \\
& \left.e_{2}^{-1} e_{3}^{-1} e_{7}\left(o_{2}\right), e_{3}^{-1} e_{7}\left(o_{2}\right), e_{7}\left(o_{2}\right), o_{2}\right)
\end{aligned}
$$

where $o_{3}=e_{7}^{-1} e_{5} e_{7}^{-1} e_{2}^{3}\left(o_{2}\right)=e_{2}^{-1} e_{5}^{-1} e_{7} e_{2}^{-1} e_{3}^{-1} e_{7}\left(o_{2}\right)$. We define $R_{3}=\left(P_{3}^{\prime}, P_{3}\right)$.

The 2-paths $R_{1}, R_{2}$, and $R_{3}$ correspond to the relations we derived in our 31 note scale from quasi-relations. These relations are close to the identity in the sense that their degree is relatively small (compared to the degrees of the possible 2arrows generated by primitive 1-arrows). We take these to be our primitive 2 -arrows.

To obtain primitive 3 -arrows and a primitive 4 -arrow, we borrow a technique from low-dimensional category theory $[2,8]$. We take as our primitive 3 -arrows $\left(R_{i} R_{j}, R_{j} R_{i}\right)$, for $i, j \in\{1,2,3\}$. We take as our primitive 4 -arrow the braid 
relation, whose source and target are

$$
\begin{aligned}
& \left(R_{1} R_{2} R_{3}, R_{1} R_{3} R_{2}, R_{3} R_{1} R_{2}, R_{3} R_{2} R_{1}\right) \\
& \left(R_{1} R_{2} R_{3}, R_{2} R_{1} R_{3}, R_{2} R_{3} R_{1}, R_{3} R_{2} R_{1}\right)
\end{aligned}
$$

respectively (note there are many recent mathematical applications of braided monoidal categories, eg. [7, 10]).

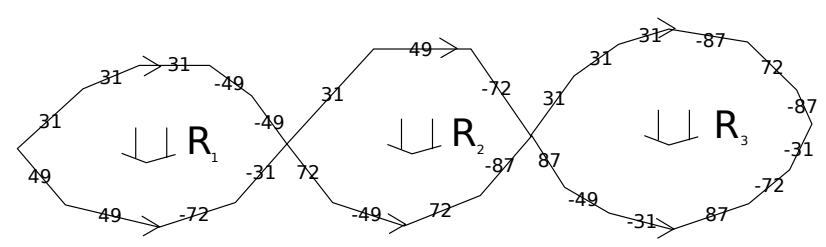

Figure 7. The 2-paths $R_{1}, R_{2}$, and $R_{3}$.

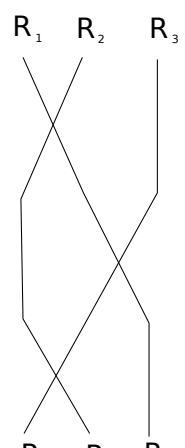

$\begin{array}{lll}\mathrm{R}_{3} & \mathrm{R}_{2} & \mathrm{R}_{1}\end{array}$

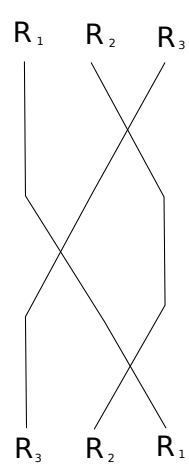

Figure 8. The braid relation.
Note that here we are abusing notation and writing $R_{i}$ for an idextension of $R_{i}$, so that each expression $R_{i} R_{j} R_{k}$ appearing in the braid relation is a 2-path whose source is $P_{3} P_{2} P_{1}$ and whose target is $P_{3}^{\prime} P_{2}^{\prime} P_{1}^{\prime}$. Our primitive 3 -arrows have relatively small degree (compared to the degrees of the possible 3 -arrows generated by primitive 2 -arrows). Our primitive 4-arrow has relatively small degree (compared to the degrees of the possible 4 -arrows generated by primitive 3 arrows).

Applying $D$ to our primitive 4-arrow gives us a sequence of frequencies. Applying $\Phi$ to this set of frequencies (with $d=0.5$ ) gives us a composition, which is an overlapping sequence of decaying sawtooth waves.

\section{Paths in Graphs}

Let us describe an ascending chromatic scale in a complicated way. Let $c \in \mathbb{R}$. For $l \in \mathbb{N}$, consider the graph $\Gamma_{l}$ whose vertices are $0,1,2, \ldots, l$ and whose edges are the pairs $\{i, i+1\}$, for $0 \leq i \leq l-1$. The geometric realisation $\left|\Gamma_{l}\right|$ can be identified with the interval $[0, l]$ so that vertex $i$ corresponds to $i \in[0, l]$. We have a map $g(l):[0, l] \rightarrow \mathbb{R}$ given by $g(l)(x)=c .2^{\frac{x}{12}}$. Consider the Hamiltonian path $\gamma_{l}$ in $\Gamma_{l}$ that begins with 0 and ends with $l$. Under $g(l)$, the vertices of $\gamma_{l}$ map to a sequence of frequencies, which upon being allocated to notes of a fixed duration, define an ascending chromatic scale. If we wish to emphasise the geometry of $\left|\Gamma_{1}\right|$, together with the special role of the subset of vertices of $\Gamma_{1}$, we can connect the successive notes of our chromatic scale with glissandi.

The compositions of this section are obtained by abstracting the above setup. Let $\Gamma$ denote a graph. Let $|\Gamma|$ denote the geometric realisation of $\Gamma$. Let $g:|\Gamma| \rightarrow \mathbb{R}^{m}$ denote a function, whose $m$ component functions are denoted $g_{i}, i=$ $1, \ldots, m$. Let $\gamma=\left(v_{0}, \ldots, v_{n}\right)$ denote a path in $\Gamma$. Let $\dot{\gamma}$ denote the path from $[0,1]$ to $|\Gamma|$ that successively, for $i=1, \ldots, n$, moves with velocity $2 n$ along the edge connecting $v_{i-1}$ to $v_{i}$, then rests at $v_{i}$ for duration $\frac{1}{2 n}$.

Suppose we are given a family $\left\{\xi_{f}\right\}_{f \in \mathbb{R}}$ of functions from $\mathbb{R}$ to $\mathbb{R}$, where $\xi_{f}$ is periodic with frequency $f$.

Let $d \in \mathbb{R}$. The path $\gamma$ determines a function $\bar{\gamma}:[0,2 n d] \rightarrow$ $\mathbb{R}^{m}$ given by $\bar{\gamma}(t)=\sum_{i=1}^{m} \xi_{g_{i}\left(\dot{\gamma}\left(\frac{t}{2 n d}\right)\right)}(t)$.

The function $\bar{\gamma}$ gives a composition generalising our ascending chromatic scale, but as it stands, it looks too general. If we wish to create music that sounds similar to music previously written, and thus appeal to the memory of the listener, we must be careful in choosing our graph $\Gamma$ and function $g$, and in making our choices refer to previous work. To do this, we use functions that are similar to functions found on existing musical instruments, and we insist that the images of the vertices of $\Gamma$ under the functions $g_{i}$ belong to subsets that have already been found to be musically useful, such as $\mathfrak{E}_{12}=\left\{440.2^{\frac{n}{12}} \mid n \in \mathbb{Z}\right\}$ or $\mathbb{Q}$.

Example 10.1. Let $E$ denote Euclidean space of dimension 2 , let $\Gamma$ denote the subgraph of the 1-skeleton of $\mathcal{Z}_{2}$ consisting of all vertices in $\{0,1,2,3\}^{2}$ and all edges between them. Let $G: E \rightarrow \mathbb{R}^{5}$ be given by $G\left(x_{1}, x_{2}\right)=$

$$
\begin{aligned}
& 440\left(2^{\frac{-45+3 x_{1}+12 x_{2}}{12}}, 2^{\frac{-33+3 x_{1}+8 x_{2}}{12}},\right. \\
& \left.2^{\frac{-26+7 x_{1}+8 x_{2}}{12}}, 2^{\frac{-21+7 x_{1}+4 x_{2}}{12}}, 2^{\frac{-17+12 x_{1}+4 x_{2}}{12}}\right) .
\end{aligned}
$$

Note that the components of $G(0,0)$ are the frequencies of major chord, the ratios $3,4,7,8,12$ all correspond to consonant intervals, and $G$ sends the vertices of $\Gamma$ to $\mathfrak{E}_{12}$. The relation between frequency and distance on a piano keyboard is approximately given by an exponential function.
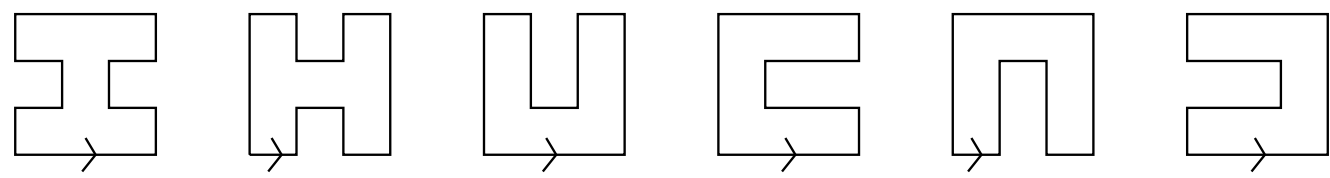

Figure 9. Hamiltonian cycles in Example 10.1, with an orientation. 
Let $g$ denote the restriction of $G$ to $\Gamma$. Our path $\gamma$ around $\Gamma$ will be a concatenation of Hamiltonian cycles based at $(0,0)$. Modulo orientation, there are six such cycles. We fix a consistent orientation of these six cycles, as in Figure 9.

To include some repetition, we insist our path $\gamma$ contains more than one Hamiltonian cycle. To limit repetition in our composition, we forbid the possibility of including two Hamiltonian cycles in $\gamma$, both of which feature an identical consecutive sequence of four vertices. This leaves us with the possibility that $\gamma$ is a concatenation of the two leftmost cycles of Figure 9. We take the leftmost one first, then the one second from the left. Upon taking $\xi_{f}(t)=\sin (2 \pi f t)$ and $d=4$, we have defined our composition $\bar{\gamma}$.

Example 10.2. Let $E$ denote Euclidean space of dimension 3 , let $\Gamma$ denote the subgraph of the 1 -skeleton of $\mathcal{Z}_{3}$ consisting of all vertices in $\{0,1,2,3,4,5,6\} \times\{0,1,2,3,4\} \times\{0,1,2,3\}$ and all edges between them. Let $G: E \rightarrow \mathbb{R}$ be given by

$$
G\left(x_{1}, x_{2}, x_{3}\right)=30 \cdot \frac{7}{7-x_{1}} \cdot \frac{5}{5-x_{2}} \cdot \frac{4}{4-x_{3}} .
$$

Let $g$ denote the restriction of $G$ to $\Gamma$. Note that the ratios $g(v) / g\left(v^{\prime}\right)$ for $v$ and $v^{\prime}$ vertices of $\Gamma$ are fractions which may be taken to have small numerator and denominator; as such sawtooth waves with frequencies given by $g(v)$ and $g\left(v^{\prime}\right)$ will commonly share harmonics of low degrees, for such pairs of vertices. The relation between frequency and distance on a violin fingerboard is given by a reciprocal function. Our path $\gamma$ in $\Gamma$ will be a Hamiltonian path, modelled as a higher dimensional relation. Let $S=\{\zeta\}$ be a one element set. Let

$$
\begin{aligned}
& \zeta_{7}=(\zeta, \zeta, \zeta, \zeta, \zeta, \zeta, \zeta), \\
& \zeta_{5}=\left(\zeta_{7}, \zeta_{7}, \zeta_{7}, \zeta_{7}, \zeta_{7}\right), \\
& \zeta_{4}=\left(\zeta_{5}, \zeta_{5}, \zeta_{5}, \zeta_{5}\right) .
\end{aligned}
$$

be a 1-path, a 2-path, and a 3-path respectively. The 0-paths in $\zeta_{7}$ naturally identify with $\{0,1,2,3,4,5,6\}$; the 1-paths in $\zeta_{5}$ naturally identify with $\{0,1,2,3,4\}$; the 2 -paths in $\zeta_{4}$ naturally identify with $\{0,1,2,3\}$. In this way the 0 -paths in $\zeta_{4}$ naturally identify with the vertices of $\Gamma$. The procedure used to define the sequence $D\left(\zeta_{7}\right)$ naturally identifies a sequence of 0 paths in $\zeta_{7}$, corresponding to a Hamiltonian path $\gamma$ in $\Gamma$. Upon taking $\xi_{f}$ to be a sawtooth wave of amplitude 1 , frequency $f$, and phase 0 , and $d=0.5$, we have defined our composition $\bar{\gamma}$.

Example 10.3. Let $E$ denote Euclidean space of dimension 3. Let $\Gamma$ denote the 1 -skeleton of $\mathcal{Z}_{3}$. Let $G: E \rightarrow \mathbb{R}$ be given by $G\left(x_{1}, x_{2}, x_{3}\right)=2^{x_{1}} 3^{x_{2}} 5^{x_{3}}$. Let $g$ denote the restriction of $G$ to $\Gamma$. Note that for vertices $v$ and $v^{\prime}$ of $\Gamma$ joined by an edge, sawtooth waves with frequencies given by $g(v)$ and $g\left(v^{\prime}\right)$ share harmonics of low degrees. Again, the relation between frequency and distance on a piano keyboard is approximately given by an exponential function.

Suppose we are given a quasi-relation $\rho=2^{a_{2}} 3^{a_{3}} 5^{a_{5}}$. Let us rearrange this expression in some way as $e=\rho^{ \pm 1}=$ $w_{n} w_{n-1} \ldots w_{2} w_{1}$ where $w_{i} \in\left\{2^{ \pm 1}, 3^{ \pm 1}, 5^{ \pm 1}\right\}$. We define a path $\gamma$ in $\Gamma$ beginning at $(1,0,2)$, whose image under $g$ is

$$
\begin{aligned}
& 50, w_{1} .50, w_{2} w_{1} .50, \ldots, e .50, w_{1} e .50, \\
& w_{2} w_{1} e .50, \ldots, e^{2} .50, w_{1} e^{2} .50, w_{2} w_{1} e^{2} .50, \ldots
\end{aligned}
$$

For a given duration $d$ and amplitude $a$, our path defines an ostinato given by applying $\Phi$ to this sequence. The ostinato repeats with a slight variation each time, due to the fact $\rho \neq 1$.

In this example we play two such ostinati at the same time, starting simultaneously, derived from the quasi-relations $2^{-4} \cdot 3^{4} \cdot 5^{-1}$ and $2^{7} \cdot 5^{-3}$. One ostinato is given by the expression $e=2^{-7} 5^{3}$ and $d=2$, and the other given by the expression $e=5^{-1} 2^{-4} 3^{4}$ and $d=\frac{160}{9}$. We allow four repetitions of the slower ostinato before termination. This corresponds to 32 repetitions of the faster ostinato.

\section{Conclusion}

We have introduced a number of different techniques involving subsets of scales in compositions constructed via similarity. Our constructions of similarity concatenation compositions involve groups, which is unsurprising since groups are a standard means of exploring similarity. Our methods involving graphs, cubist sets, and higher dimensional relations have a topological and combinatorial flavour. This too should not be surprising: musical data is often expressed combinatorially as sequences of integers or real numbers, and associations are frequently expressed as higher dimensional relations. It makes sense to explore these ideas in 31 tone equal temperament, as well as 12 tone equal temperament.

\section{References}

[1] J. C. Baez and J. Dolan. Categorification. Contemporary Mathematics 230, pp. 1-36, 1998.

[2] J. C. Baez and M. Neuchl. Higher Dimensional Algebra I: Braided Monoidal 2-Categories. Advances in Mathematics 121, pp. 196-244, 1996.

[3] D. J. Benson. Music: A Mathematical Offering. Cambridge University Press, November 2006.

[4] A. Chan. Yoneda algebras of quasi-hereditary algebras, and simple-minded systems of triangulated categories. PhD Thesis. University of Aberdeen, Scotland, UK, 2014.

[5] J. Chuang and R. Rouquier. Derived equivalences for symmetric groups and $s l_{2}$-categorification. Annals of Math. 167, pp. 245-298, 2008.

[6] J. Chuang and W. Turner. Cubist algebras. Advances in Mathematics 217 (4), pp. 1614-1670.

[7] Z. Dell. A Characterization of Braided Enriched Monoidal Categories. arxiv 2104. 07747, 2021. 
[8] A. Joyal and R. Street. Braided tensor categories. Advances in Mathematics 102, pp. 20-78, 1993.

[9] D. Huron. Sweet Anticipation. Music and the Psychology of Expectation. Bradford, 2008.

[10] C. Jones, D. Penneys, D. Reutter. A 3-categorical perspective on G-crossed braided categories. arxiv 2009. 00405, 2020.

[11] C. Huygens. Brief betreffende de harmonische cyclus. Histoire des Ouvrages des Sçavans, Rotterdam, pp. 7888, October 1691.

[12] A. Manion and R. Rouquier. Higher representations and cornered Heegaard Floer homology. arxiv 2009. 09627, 2020 .

[13] W. Turner. Algebraic pure tone compositions constructed via similarity. Examples 1 and 2. World Journal of Education and Humanities. 2, 3, p. 21-27. http://homepages.abdn.ac.uk/w.turner/pages/

[14] W. Turner. Some scales that are similar to the chromatic scale. Far East Journal of Mathematical Sciences 122, 2, p. 199-214. http://homepages.abdn.ac.uk/w.turner/pages/

[15] W. Turner. Subsets of scales in compositions constructed by similarity. Example 1, 2, 3, 4, 5, 6, 7, 8 . http://homepages.abdn.ac.uk/w.turner/pages/ 